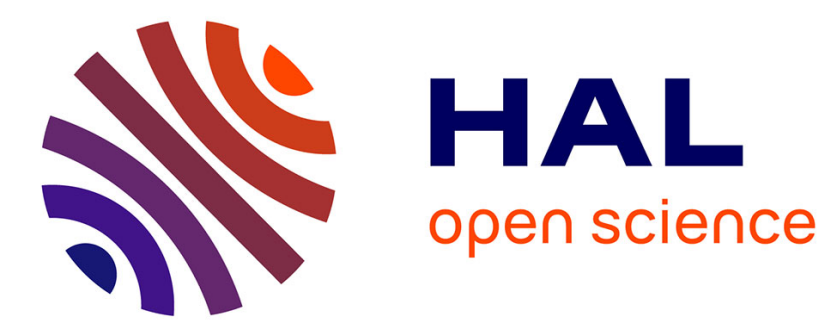

\title{
Une évolution dans la narration enfantine, le dit, le redit et le non-redit
}

Regine Delamotte

\section{To cite this version:}

Regine Delamotte. Une évolution dans la narration enfantine, le dit, le redit et le non-redit. Théories didactiques de la lecture et de l'écriture, Presses universitaires du Septentrion, 2017. hal-02375583

HAL Id: hal-02375583

https://hal-normandie-univ.archives-ouvertes.fr/hal-02375583

Submitted on 22 Nov 2019

HAL is a multi-disciplinary open access archive for the deposit and dissemination of scientific research documents, whether they are published or not. The documents may come from teaching and research institutions in France or abroad, or from public or private research centers.
L'archive ouverte pluridisciplinaire HAL, est destinée au dépôt et à la diffusion de documents scientifiques de niveau recherche, publiés ou non, émanant des établissements d'enseignement et de recherche français ou étrangers, des laboratoires publics ou privés. 


\section{Régine Delamotte, «Une évolution dans la narration enfantine : Le dit, le redit et le non- redit ", Théories didactiques de la lecture et de l'écriture, Cora Cohen-Azria \& Ana Dias-Chiaruttini (dirs), Le Septentrion}

\section{UNE EVOLUTION DANS LA NARRATION ENFANTINE : LE DIT, LE REDIT ET LE NON-REDIT}

\section{Introduction/positionnement}

Pour continuer à débattre avec Yves Reuter sur le récit chez l'enfant, il sera question de deux narrations enfantines orales d'une petite fille, à 3 ans 6 mois et à 4 ans 6 mois, à partir d'une histoire, L'anniversaire de Babar. Il s'agit avant tout pour l'enfant du plaisir de raconter en feuilletant l'album avec sa mère. Cette envie de dire et de redire cette même histoire s'est présentée de nombreuses fois sur une durée de plus de 2 ans. Les enfants vivent, souvent sur de longues périodes, en compagnie de leurs histoires privilégiées (Nalouti, 1998). Entre ses deux narrations, l'enfant a grandi, développé son langage et fait l'expérience de la petite puis de la moyenne section de maternelle. Ce parcours dans le récit enfantin, son évolution et les interrogations qu'elle suscite constituent la matière de cette contribution.

L'analyse présentée s'inscrit dans un cadre théorique que j'ai développé par ailleurs (entre autres, Delamotte, 1997, 2004, 2012). Je voudrais, brièvement, rappeler que l'appropriation du langage par les enfants se réalise dans des contextes variés, dans lesquels ils rencontrent une grande diversité de manières de faire selon les lieux, les personnes, les enjeux. Cependant, toute appropriation d'une pratique langagière a besoin de modèles et de personnes porteuses de ceux-ci auxquelles les enfants ont envie de s'identifier. L'activité narrative se distingue par un certain nombre de spécificités de contenu et de forme de discours (personnages, événements, temporalité, connecteurs, dramatisation, évaluation, etc.). Elle se signale donc par des invariants qui marquent son appartenance générique, bien étudiés en narratologie (Reuter, 2000, 2005). Mais il y a une différence entre reconnaître un genre discursif par des marquages récurrents et prévisibles et réduire l'ensemble de ses usages à cette récurrence et à cette prévisibilité. Diversité langagière et modèles langagiers vont de pair, car il y a de multiples façons possibles de raconter des histoires, même si raconter relève de savoir-faire bien identifiables. L'activité narrative n'existe que parce que des récits circulent entre les personnes sous des formes toujours reproduites et toujours réinventées. Ce que rencontrent donc les enfants, ce ne sont pas des modèles de langage, mais des personnes bien réelles dans leur reprise-modification des manières de dire (François, 1993). A travers ces rencontres, les enfants se confrontent à l'infinie richesse de la parole et à eux-mêmes dans le langage (Morgenstern, 2009).

La présente contribution tient compte du fait qu'il s'agit, dans le cas étudié, d'une relation intersubjective dans laquelle la mère et sa fille adoptent diverses positions, marquant leur présence par l'affirmation de points de vue. Leurs manifestations verbales et non-verbales permettent de moduler ce 
qu'elles disent tout en faisant connaître ce qu'elles sont. Le dialogue constitue aussi pour elles un espace dans lequel se construit une référence commune du monde au-delà d'un savoir partagé du récit. Sans oublier, enfin, que tout moment dialogique de ce type est pris dans une histoire relationnelle, singulière, vivante, sur un temps long, dont ne peut identifier, hic et nunc, les traces, mais dont on est tenu de faire l'hypothèse de leur présence.

\section{Problématique et questionnements}

Lors de l'appropriation du langage par l'enfant, du récit en l'occurrence, un certain nombre de maniements lui sont enseignés. Par exemple, les marques de cohérence par le maintien d'une continuité lexicale ou celles de cohésion par l'usage des anaphores. Leur maîtrise exige de longs apprentissages. Mais il y en a d'autres qui relèvent d'une culture, au sens très large, et qui font appel à d'autres dimensions de l'activité narrative. "L'enfant sait faire des récits intéressants avant de savoir faire des récits cohésifs-cohérents » (François, 1990, 26), « intéressants » signifiant, pour François, qui sont source de plaisir partagé, de surprise, de découverte par l'enfant du statut de narrateur et de redécouverte par l'adulte de ce qu'est être un enfant qui raconte.

Mon propos n'a ainsi pas d'objectif d'évaluation. Il vise d'abord une description, puis une tentative d'interprétation. L'aspect évaluatif a été maintes fois étudié à l'aide de grilles diverses d'analyse. La grande difficulté concerne la généralisation des résultats. Une prudente évaluation de la conduite du récit chez le jeune enfant ne peut se faire qu'en lien avec une situation narrative ciblée et non pour l'activité narrative dans son ensemble (Salagnac, 2007). Plus précisément, en fonction de l'âge de l'enfant et des enjeux de la situation, «....il n'y a pas à privilégier ce qui doit être dit par rapport à ce qui peut être dit » (François, 2004, 80). Cette option est à la base d'une étude en cours des récits de Jeanne dont la présente contribution constitue une première version ne traitant que de quelques aspects ${ }^{1}$.

On le sait, toute activité langagière témoigne à la fois des savoir-faire du sujet et de son rapport à cette activité. Lorsque nous écoutons la petite Jeanne raconter et raconter encore l'anniversaire de Babar, nous découvrons non seulement des capacités de mise en mots du récit, mais aussi une relation particulière à cette histoire, comme histoire et comme moment partagé. Jeanne offre à notre écoute son vécu de l'histoire, celui d'une très jeune narratrice qui raconte parce que c'est pour elle l'occasion renouvelée de dire et de se dire dans une relation privilégiée avec sa mère dont elle connaît le plaisir à l'écouter.

On le sait aussi, entre 3 ans et 6 ans, l'activité narrative des enfants ne peut se réaliser qu'en interaction avec des interlocuteurs plus experts. L'autonomie dans la conduite d'un récit n'est pas à la

\footnotetext{
${ }^{1}$ Régine Delamotte et Anne-Marie Clinquart, De l'enchevêtrement des modalités narratives au processus normé de la restitution d'une histoire : les récits de Jeanne entre 3 et 4 ans (rédaction en cours).
} 
portée de très jeunes enfants. La question du rapport à l'activité langagière se pose donc aussi pour l'adulte qui écoute l'enfant et qui l'aide à dire. On retrouve, à nouveau, la question du point de vue en faisant l'hypothèse de différences entre l'enfant et l'adulte, au-delà de la dimension affective et du plaisir partagé. C'est toute la conception de ce qu'est « raconter une histoire déjà connue », de part et d'autre, qui est en jeu (qui est l'enjeu ?). Qu'en pense l'enfant? Qu'en pense l'adulte ? Quels sont les effets en discours de ces différences ? Quel impact sur la socialisation de l'enfant?

Des questions s'imposent donc quant à ces différences. Du côté de l'adulte, engagé dans l'échange, en quoi consiste sa participation ? Du côté de l'enfant, que restitue-t-il ou non du texte de départ (le redit) ? Qu'est-ce qui mérite ou non, de son point de vue, d'être retenu étant donné que tout ne peut être restitué (le non-redit)? Quelle place est laissée ou non au commentaire, qui peut être interne ou externe au récit (le dit)? Ces questions seront posées aux deux corpora, la comparaison entre eux pouvant donner des indications sur l'évolution d'une pratique narrative, parmi d'autres, pour Jeanne et suggérer une réflexion plus générale.

\section{Corpora}

\subsection{Le contexte de production}

Je ne fais que signaler rapidement que les échanges analysés ici se déroulent dans une culture de l'écrit historiquement et spatialement située, dans un milieu social favorisé du point de vue culturel et dans une habitude de relations parents/enfants donnant toute sa place à la prise de parole enfantine. Les lectures partagées constituent donc une pratique hautement valorisée dans l'environnement de Jeanne (Grossmann, 1996, Freier et alii, 2005). Derniers points, et non les moindres : la mère est professeure des écoles et elle a longtemps exercé en maternelle; elle est docteure en Sciences du Langage ${ }^{2}$.

L'expérience langagière de Jeanne relève, plus généralement, d'une pratique sociale répandue qui entre dans un rituel, lui-même très répandu :

- un contexte familial, ordinaire, quotidien pour une activité répétitive et souhaitée ;

- une histoire racontée à l'enfant et restituée par elle avec un album comme support. A la différence d'un face à face uniquement verbal, les regards se portent tour à tour de l'album à l'interlocutrice et l'activité enchevêtre faire et dire ;

- une même dyade interlocutive (la mère et l'enfant), chaque interlocuteur étant, tour à tour, narrateur et auditeur, selon les moments de lecture partagée. Le texte original a ainsi été lu par sa mère, in extenso et sans variation lexicale, plusieurs fois à l'enfant et à sa demande.

\footnotetext{
${ }^{2}$ Anne-Marie Clinquart (1997) La reformulation dans des situations interactives contrastées, Septentrion, Thèse à la carte.
} 


\subsection{Les deux enregistrements}

Ils ont été réalisés par la mère. Ils n'avaient à l'origine aucun objectif de recherche. L'histoire étant souvent réclamée par Jeanne depuis qu'elle a 3 ans, sa mère a eu l'idée d'enregistrer une restitution à 3 ans 6 mois pour en garder un souvenir. C'est ainsi qu'elle a présenté à Jeanne la présence de l'appareil d'enregistrement. Cette histoire étant réclamée à nouveau, un an plus tard, parce que l'album a refait une apparition, la mère a réalisé un second enregistrement dans des conditions identiques au premier. C'est une chance pour la recherche qui n'a pas toujours à sa disposition de telles productions «spontanées » d'enfants totalement hors contexte scientifique. Les données que je vais analyser sont ainsi constituées par deux corpora, produits à un an de distance (désormais $\mathrm{C} 1$ et C2).

Le matériau dont je dispose est sonore. Le filmage aurait été plus qu'intéressant pour une analyse détaillée de la corporéité et de la gestualité de Jeanne, comme celles de sa mère. Les éléments de continuité et de discontinuité en dialogue tiennent en partie à cette dimension de l'interaction (Garitte, 1998). Et, surtout, le filmage aurait permis de noter la manière dont l'enfant manipule l'album avec sa mère. J'ai pu, cependant, avoir des éléments d'information sur ce point en interrogeant la mère qui, tout au long de l'écoute et de la réécoute de l'enregistrement, a retrouvé des souvenirs assez précis. Ils expliquent, en effet, une part non négligeable de ce qui est entendu. Par exemple, lorsqu'en $\mathrm{C} 1$, Jeanne produit un discours qui n'a rien à voir avec la fin de l'histoire. On comprend mieux son intervention si l'on sait qu'elle commente la $3^{\text {ème }}$ de couverture, après un « alors, voilà » qui clôt son récit :

Jeanne : alors voilà /// et ça c'est qui ? / peut-être que c'est Babar qui s'est déshabillé / peutêtre que c'est Cornélius qui s'est déshabillé / peut-être que c'est peut-être que c'est Podular qui s'est déshabillé / de toute façon on voit que c'est un éléphant / c'est un éléphant qui s'est déshabillé / qui va offrir sa peau à quelqu'un (C1)

\section{Outils d'analyse et premières investigations}

L'analyse vise une étude comparative permettant de mettre en lumière la manière dont les modalités de l'activité narrative se modifient en fonction de l'âge selon les trois questions retenues dans la problématique. Pour un amorçage de l'analyse, j'ai eu recours à des « comptages », légitimés par des écarts très nets entre les deux récits. Mais l'essentiel de l'analyse est de nature qualitative.

\subsection{La complémentarité du quantitatif et du qualitatif}

Du point de vue quantitatif, la difficulté est de choisir les unités à prendre en compte. La segmentation peut s'effectuer de diverses manières, une bonne mesure pouvant être trouvée en ajustant 
au mieux à la problématique. Concernant l'activité narrative, F. François $(1997,87)$ propose de partir des «saynètes » caractérisées par un contenu dominant ou un genre dominant, puis d'interroger les prises de «paroles liées » au sein des échanges (question/réponse, accord/désaccord, etc.) et, enfin, de tenter d'identifier, au sein des interventions de chaque interlocuteur, des «paquets de mises en mots » d'un ou plusieurs syntagmes référant de manière cohérente à des objets présents ou absents. C'est à ce niveau que j'ai situé mon analyse quantitative, désignant ces paquets par le terme d' « énoncés ».

Du point de vue qualitatif, s'agissant de la prise de parole de l'enfant, se dégagent deux ensembles. Un ensemble d'énoncés narratifs qui renvoie au récit. Un ensemble d'énoncés hors récit, discursivement variés : quelques questionnements et surtout des commentaires. Notons que ce hors récit, non partagé, de la production enfantine donne une plus grande liberté de création à l'enfant et, du fait du non partage, une moindre possibilité d'étayage de l'adulte qui viserait le strict déroulement de l'histoire.

Je vais donner, d'abord, quelques résultats concernant la participation de la mère, puis ceux concernant les récits de Jeanne, en visant invariants et variations. Mon objectif, en effet, est d'identifier et de décrire ce qui varie ou non entre les deux corpora, indépendamment des raisons qui l'expliquent, sauf si elles s'imposent. Mais la plupart échappe à l'analyse, trop de paramètres non maîtrisables entrant en jeu. Au mieux, on peut se livrer à des interprétations hypothétiques dont la confirmation exigerait d'autres données.

\subsection{Les interventions de la mère}

S'agissant de l'adulte, la question se pose des possibles effets de ses interventions sur la sélection des contenus et la mise en récit de l'enfant. Il est heureux que j'aie pu avoir les commentaires de la mère sur ces échanges, car la mimogestualité y a une place centrale. Et surtout, étant donné le peu d'interventions verbales de la mère, cette mimogestualité s'avère fondamentale pour le maintien de l'interaction et l'occupation de l'espace dialogique par l'enfant.

En effet, la mère se manifeste de manière permanente auprès de l'enfant par des hochements de tête, des sourires, des regards, etc. L'enfant, de son côté, est attentif à ces manifestations et son regard va régulièrement de l'album au visage de sa mère, quitte même à lui faire remarquer qu'elle manque parfois d'attention. Verbalement, on peut faire état de deux postures chez la mère.

En C1, l'enfant ne manifestant aucun désir de « coller » au texte, la mère l'aide simplement à conduire son propre discours. Elle ne produit, ainsi, que 8 très brèves interventions, soit 9 énoncés en tout, face aux 126 énoncés de l'enfant :

- un « ah bon? » dubitatif

- une réponse à la demande de Jeanne : « qu'est-ce qu'i dit Babar, hein ?»

- trois échanges concernant le sens des mots ou expressions (soit six interventions) 
En $\mathrm{C} 2$, en revanche, l'enfant tenant à tout prix à rester au plus près du texte, la mère ne répond qu'à ses sollicitations pour le faire. Face aux 86 énoncés de Jeanne, il s'agit seulement de 3 interventions ne représentant que 2 énoncés et un mot, tirés du texte original et soufflés à l'enfant.

\section{Les récits de Jeanne}

Je m'en tiendrai à deux aspects : ce que Jeanne redit et ne redit pas de l'histoire et ce que jeanne dit en général dans les deux échanges.

\subsection{La narration de Jeanne face au texte original}

Quelques chiffres me permettent de donner des tendances générales. J'ai identifié, au fur et à mesure du déroulement de l'interaction, ceux des énoncés de Jeanne renvoyant directement à l'histoire. On peut ainsi constater que :

- en C1, 35\% de l'histoire de départ est présente (contre $65 \%$ absente)

- en C2, 68\% de l'histoire de départ est présente (contre 32\% absente)

Cette inversion constatée, l'important est d'identifier la matière du redit, à savoir ce qui a, du point de vue de l'enfant, mérité d'être repris ou non. Il est apparu intéressant d'examiner cette matière sous les deux aspects du contenu significatif et de la formulation linguistique.

Tout récit présente, globalement, des contenus obligatoires et d'autres facultatifs pour la cohérence de l'histoire, même si, pour un récit particulier, on peut discuter de ce qui relève de ces catégories.

Pour les récits de Jeanne, l'examen montre que le non-redit en C1, étant donné son importance, porte sur tous types de contenus, de structuration ou de détail. Il concerne parfois des blocs du récit équivalents à des saynètes. En C2, il ne concerne que des détails du récit, des «trous » dans l'histoire correspondant à des énoncés et qui, n'en modifiant pas la construction générale, seraient abandonnés dans un résumé. Cependant, un ensemble important et identique d'aspects facultatifs se trouve non retenu dans les deux récits.

J'en donne deux exemples : les segments en droit du texte original (désormais TO) ne sont pas repris ${ }^{3}$.

TO : «Alors ils coupent des arbres et dressent un échafaudage contre la montagne... »

$\mathrm{C} 1$ : alors alors $i$ construit des échafaudages

$\mathrm{C} 2$ : ils dressent un échafaudage contre la montagne

TO : «Cornélius rassemble les musiciens de la garde royale. Un, deux, trois, les trompettes sonnent la fanfare...»

\footnotetext{
${ }^{3}$ Les extraits du TO sont entre guillemets, contrairement aux extraits des récits de Jeanne.
} 


\section{$\mathrm{C} 1$ : un deux trois i font / les trompettes sonnent la fanfare \\ $\mathrm{C} 2$ : et un deux trois / les trompettes sonnent la fanfare}

Un point commun aux deux récits, concernant le non redit, mérite d'être signalé. Les mises en mots des états mentaux des personnages ne sont pas reprises.

En voici quelques exemples. Mais cet aspect serait à travailler dans le cadre de la problématique des théories de l'esprit chez l'enfant que je ne peux aborder ici (Plumet, 2008).

En droit, on voit les segments non repris du $\mathrm{TO}$ en $\mathrm{C} 1$ comme en $\mathrm{C} 2$ :

«Comme il l'a promis, le singe Zéphir regarde... »

«Cher Podular, je voudrais faire une surprise à Babar pour son anniversaire. J'ai une idée, pouvez-vous sculpter dans la montagne une statue géante... »

«Podular, inquiet, rappelle les deux marabouts...»

«Le plus difficile est fait, pense Podular qui s'applique pour que la cravate soit jolie... »

«Pendant ce temps à Célesteville, tout le monde est préoccupé, l'anniversaire de Babar doit être une belle fête... »

«Zéphir saute avec décision dans l'auto rouge... »

«Dans les cuisines du palais, la Vieille Dame et Céleste sont venues goûter les crèmes. Aucun doute, les gâteaux seront délicieux. Babar se promène d'un air songeur. "Se doute-t-il de quelque chose », pense Céleste inquiète...»

«Dans leur joie, ils sautent comme des fous... »

Du point de vue linguistique, on repère la mise à l'écart de certaines formulations (en droit dans les exemples) qui vont rejoindre le facultatif du récit. En voici quelques exemples :

«Ils trouvent l'endroit qui convient le mieux pour sculpter la pierre...» (TO)

Ils trouvent l'endroit pour sculpter la pierre ( $\mathrm{C} 1$ et $\mathrm{C} 2)$

« Soyez gentils, montez la garde pour que je puisse travailler tranquillement... (TO)

Soyez gentils que je puisse travailler tranquillement... » (C1 et $\mathrm{C} 2)$

«Podular est en train de sculpter l'œil de Babar avec son marteau pneumatique... » (TO)

Podular est en train de sculpter l'œil de Babar (C1 et $\mathrm{C} 2)$

Dernier point à signaler : entre la question de la reprise des contenus et celle de la reprise des mises en mots, se situe la question de la reprise reformulée ${ }^{4}$. Dans ce cas, si le contenu est conservé, la mise en mots subit divers types de transformations à la mesure du rapport au monde de l'enfant et de ses moyens linguistiques.

\footnotetext{
${ }^{4}$ Je ne développe pas ce point qui est en train d'être travaillé de manière très détaillée par Anne-Marie Clinquart qui en a l'expertise linguistique.
} 
Je donne deux exemples d'ajouts (en droit), par rapport au TO, qui peuvent être interprétés comme des commentaires propres à la culture enfantine : " c'est pas grave ! », " c'est un cadeau ! ", et un exemple de reformulation: " $t$ 'inquiète pas!» qui renvoie à une manière de dire fréquente au quotidien :

Oh merci messieurs s'exclame Babar / c'est pas grave je la recollerai (C1)

Merci messieurs! j'aime vraiment cette pipe! ce n'est pas grave je la recollerai (C2)

On peut remarquer, au passage, l'évolution de la mise en mots, avec la mémorisation de «j'aime vraiment cette pipe » et la double négation comme norme.

Mais attention ajoute Céleste / Babar ne doit rien savoir / c'est un cadeau ! (C1)

Mais attention ajoute Céleste / Babar ne doit rien savoir / c'est un cadeau ! (C2)

«Mais Podular le rassure : «Pas du tout, Arthur, le secret est bien gardé. Ton ami Zéphir n'est qu'un taquin. » (TO)

Mais non t'inquiète pas / dit Podular / ton ami Zéphir n'est un n'est qu'un taquin... (C1)

Au niveau du choix des unités linguistiques, des cas de reformulation peuvent, plus facilement que d'autres cas de variation, s'expliquer soit par l'usage enfantin du langage, soit par la forme orale du récit face au texte écrit de l'album. On peut penser que les écarts par rapport à l'énoncé original sont pour beaucoup la traduction de la difficulté à transposer dans l'oralité des formes écrites.

- le passage du «nous » au « on » :

« Nous voulons construire notre nid sur cette montagne!» (TO)

Oh! on veut faire notre nid sur cette montagne $(\mathrm{C} 1)$

Oh! on veut faire notre nid sur cette montagne $(\mathrm{C} 2)$

- la généralisation de « faire »:

«Pouvez-vous sculpter dans la montagne une statue géante du roi? » (TO)

Vous pouvez faire une géante statue dans la montagne? (C1)

«Il descend pour sculpter les pieds. » (TO)

Pendant que Podular est en train de faire le pied de Babar... (C2)

"Podular va tailler dans le rocher une grande statue de Babar » (TO)

Podular va faire une grande statue de Babar (C1 et $\mathrm{C} 2)$

- la généralisation de « dire » :

«Nous avons bien avancé, n'est-ce pas ? lui crie Zéphir.» (TO) 
Nous avons bien avancé n'est-ce pas dit Zéphir (C1)

«Oh!oui, vite! », ajoute Arthur, en parlant un peu du nez » (TO)

Oh oui oui dit Arthur en parlant un peu du nez (C2)

\subsection{Le hors-récit de la narration de Jeanne}

Oublions les énoncés du texte de départ et examinons comment se répartissent les énoncés dans les deux récits de l'enfant. Le calcul concerne donc la part de présence du récit d'origine par rapport à l'ensemble de ce qui est produit (le dit) dans ses récits par l'enfant.

On peut noter :

- $\quad$ en $\mathrm{C} 1,34 \%$ de récit pour $66 \%$ de commentaires

- en $\mathrm{C} 2,96 \%$ de récit pour $4 \%$ de commentaires

Ce que j'appelle « commentaires » présente en fait deux types de mises en mots différents : des questionnements et des commentaires, mais les premiers ont une présence minime par rapport aux seconds.

Le questionnement s'adresse essentiellement à la mère. Jeanne prend à partie sa partenaire pour qu'elle l'aider à dire. Elle demande aussi qu'elle lui explique des points particuliers. Elle la pousse à donner son point de vue et, du coup, à se donner l'occasion d'opposer le sien ou de le négocier.

Je ne prends que des exemples en $\mathrm{C} 1$, puisqu'en $\mathrm{C} 2$ la part du hors-récit est très faible. On trouve, par exemple des questions :

- sur l'histoire :

Jeanne : pourquoi i veulent pas qu'on les voyent / euh Pom et Alexandre / et puis Flore hein?

Jeanne : elle s'est fait mal où Flore hein? (C1)

Jeanne : qu'est-ce qu'i dit Babar hein? (C1)

- sur l'explicitation d'un terme :

Jeanne : ça c'est un / ça c'est un saxophone / ça c'est des trompettes et ça c'est un saxophone / ça c'est quoi comme instrument?

Mère : un cor

Jeanne : un cor / un accordéon / ça ressemble à un accordéon

Mère : ah non

Jeanne : oui mais un cor c'est un accordéon

Mère : mais non

Jeanne : mais si un cor un accordéon...» (C1) 
Mais le questionnement peut aussi s'adresser aux personnages de l'histoire. Le support de l'illustration est ici incontournable, l'enfant s'adressant à l'image du personnage.

Jeanne : quand même quand même quand même / là il y a une seule bougie / t'as pas un an Babar! / alors pourquoi y a une seule bougie ? / là là yen a pas / là yen a beaucoup / là yen a qu'une seule et là yen a pas / qu'est-ce que c'est que ça? / qu'est-ce que c'est que ce gâteau? I qu'est-ce que c'est que ce gâteau madame Céleste et monsieur Babar? (C1)

Les commentaires sont particulièrement fréquents et leur diversité tient à des types de référence différents. Je m'appuie ici sur une définition très générale pour laquelle parler de référence, c'est s'intéresser aux différentes façons que le discours a de renvoyer à autre chose que lui-même. Ce à quoi le discours réfère peut être présent ou absent, réel ou virtuel (Hudelot, 1989). Les possibilités enfantines dans le domaine de la référence s'avèrent très larges. Je m'en tiens à quelques exemples du discours de Jeanne, en soulignant que certaines catégories de référence se combinent dans un même énoncé. On trouve ainsi différents types de commentaires.

Des commentaires sur des objets présents, apparemment hors activité narrative, mais qui peuvent être en relation lointaine avec elle lorsqu'ils portent sur un inconfort semblant gêner Jeanne pour raconter.

Jeanne : $i$ va être trop petit ce pyjama regarde comme j'ai grandi maman / je peux même plus le redescendre il remonte / oh là là t'as vu je peux même plus le redescendre ce pyjama... (C1)

Jeanne : t'as vu i griffe mon petit ongle on va le couper / i faut pas avoir des longs des des des des longs doigts de pieds comme ça / sinon i vont trouer les chaussettes $(\mathrm{C} 1)$

Le second exemple semble plus éloigné de l'activité narrative que le premier, mais il concerne aussi le fait d'avoir grandi. C'est sans doute extrapoler bien au-delà du démontrable, cependant, la manière qu'a Jeanne de prendre en charge son discours face à sa mère va bien dans le sens de la conquête d'un statut de « grande ».

Des commentaires sur la situation d'énonciation comme forme du maintien de l'interaction, puisqu'ils pointent un laisser-aller de la mère ou un manque de réactivité de sa part :

Jeanne : (la mère regarde l'album allongée sur le lit et a tendance à fermer les yeux !) tu peux te coucher sans fermer les yeux hein sinon tu vois rien / si tu fermes les yeux et ben tu vois rien / garde les yeux ouverts sinon tu vois rien... $(\mathrm{C} 1-4) \ldots$ tu vois rien maman (C1 - 9)... mais tu vois rien maman / tourne les yeux tourne les yeux tu vois rien (C1 - 10) Jeanne : (la mère ralentit le récit en ne tournant pas la page assez vite) tu vois i fallait tourner la page hein (C1 - 13) 
Des commentaires sur les illustrations qui constituent des pauses au sein de l'activité narrative. Ils sont souvent ponctués de longs silences, pendant lesquels Jeanne observe l'image, comme coupée de la nécessité interactive, comme si elle se parlait à elle-même Certains occupent une fonction purement descriptive, d'autres plus descriptive/explicative.

Jeanne : elle est toute rose toute rose Flore / elle a une robe rose des chaussures roses toute rose / et toute rose et grise... (C1)

Jeanne : c'est peut-être parce qu'il a tellement remué sa trompe que voilà / ça peut être ça aussi / ça peut être aussi que i a trop remué sa trompe / qu'elle devient toute froissée / que les autres $i$ zont pas leur trompe comme eux $(\mathrm{C} 1)$

Beaucoup de commentaires, affichant une fonction explicitement phatique de maintien du lien avec l'adulte, restent insérés dans le cours du récit et ne donnent pas lieu à des pauses.

Jeanne : t'as vu il est là Babar / t'as vu les bébés $i$ sont dans leur poussette (C1)

Jeanne : t'as vu $i$ i fait comme ça avec sa patte / et puis i tient avec sa trompe en même temps / comme ça $i$ fait $(\mathrm{C} 1)$

Jeanne : alors t'as vu là i font tous les / i font la la grande roue les / dis donc yen a beaucoup hein / yen a qui sont pas les enfants de Céleste et de Babar / regarde yen a beaucoup / yen a yen / yen a pas qui sont les enfants de Céleste et de Babar $(\mathrm{C} 1)$

Jeanne : ah t'as vu $i$ sont tous les éléphants / elle se trompe Céleste regarde à qui elle le donne / il est là Zéphir / tu le vois là Zéphir?

Des commentaires sur la connaissance du monde renvoient à des pratiques diverses de décontextualisation, généralisation, définition, recommandation. Comme si raconter était aussi pour Jeanne l'occasion de faire la preuve de ce qu'elle sait du monde qui l'entoure, ou de la façon dont elle l'appréhende. Dans les exemples qui suivent, Jeanne définit et produit des normes d'expérience et d'usage. On notera la répétition de «normalement » et de « il faut ».

Jeanne : les marabouts c'est des oiseaux (C1)

Jeanne: normalement les éléphants $i$ marchent comme ça / et on voit pas leur queue les éléphants normalement i zont des queues $(\mathrm{C} 1)$

Jeanne : des échafaudages i faut mettre des bois / alors ces échafaudages i sont pas solides / c'est pas solide hein / i faut pas sauter sur les échafaudages (C1)

Certains commentaires concernent des négociations du rapport au monde par le biais du choix des désignations. Dans ce cas de figure, on voit combien l'adulte cède devant la logique enfantine pour ne pas rompre l'élan du récit. 
Jeanne : et là c'est Zéphir / c'est l'enfant de Céleste et Babar aussi

Mère : mais non c'est un singe

Jeanne : oui mais même si c'est un singe

Mère : les éléphants ne font pas de singe

Jeanne : ouais / mais Zéphir il est dans cette famille quand même

Mère : oh oui

Jeanne : il est dans la famille des éléphants (C1)

Jeanne : les éléphants i font du bruit / $i$ sifflent avec leur trompe

Mère : on dit ils barrissent

Jeanne : oui mais on peut dire i sifflent aussi avec leur trompe

Mère : $m m(\mathrm{C} 1)$

Des commentaires sur la référence à d'autres histoires mettent implicitement en scène les événements qui ont précédé l'acte d'énonciation, notamment les échanges qu'ont pu avoir auparavant les interlocutrices. Pour exemples, le partage de connaissances communes autour d'autres histoires lues.

Jeanne : Cornélius des fois i fait des ptites bêtises hein / i s'assoit dans son chapeau / il est coquin aussi hein / Cornélius tu feras plus ça? (C1)

(référence à une autre histoire de Babar)

Jeanne : les éléphants i font $d u$ bruit / $i$ sifflent avec leur trompe

Mère : on dit ils barrissent

Jeanne : oui mais on peut dire i sifflent aussi avec leur trompe

Mère : $m m$

Jeanne : comme le cochon qui siffle comme Muguet / Muguet i siffle / d'habitude les cochons $i$ font RRRRR... (C1)

(référence à une autre histoire : «Le petit Cochon qui sifflait»)

\section{Discussion/conclusion}

L'analyse des deux récits de Jeanne laisse apparaître quelques éléments saillants. D'abord, qu'il n’y a pas de réelle évolution d'un récit polygéré vers un récit monogéré. Il s'agit ici d'une évolution entre dire et redire, le non-redit changeant totalement de statut. La mère, en effet, intervient très peu verbalement, mais marque toujours une présence attentive qui suffit à Jeanne pour occuper l'espace du dialogue. L'autonomie verbale de l'enfant est, de plus, rendue possible par le support de l'album et la fréquence de sa lecture partagée avec la mère. Ensuite, la différence n'est pas non plus significative sur le plan linguistique : bagage lexical, diversité énonciative, outils discursifs, etc. sont à peu de 
chose près équivalents. On sait que, de ce point de vue, entre 3 et 4 ans, les différences entre enfants du même âge sont plus grandes que les différences entre groupes d'âges. On peut, cependant, remarquer, au niveau textuel, une modification de l'ordre événementiel plus importante en $\mathrm{C} 1$ qu'en C2. Elle s'explique plus par les retours en arrière sur les images, plus fréquents en $\mathrm{C} 1$ qu'en $\mathrm{C} 2$, et par la part très importante des commentaires en $\mathrm{C} 1$ que par une possible différence de maitrise de l'organisation du récit. Les corpora en l'état ne permettent pas de conclure sur ce point. Ils permettent simplement de noter qu'en C1 l'attention de Jeanne se porte essentiellement sur l'image et en C2 sur le texte. En $\mathrm{C} 1$, Jeanne cherche à comprendre la logique de la relation entre les images et le texte et donne son point de vue sur cette relation. En C2, elle prend appui sur les images pour restituer au mieux le texte original. La question de la mémorisation du texte est plus une réalité en $\mathrm{C} 2$ qu'en $\mathrm{C} 1$. Mais, dans les deux cas, Jeanne a mémorisé une importante partie du texte de départ.

C'est dire combien la différence entre les deux récits se situe, avant tout, dans le traitement de l'histoire et dans la signification donnée à l'activité narrative par Jeanne, sa mère suivant sans rien imposer le mouvement de l'enfant, lui laissant occuper la place énonciative qu'elle souhaite. A 3 ans, pour Jeanne, raconter c'est prendre prétexte de l'histoire pour dire des choses à propos de ce qu'elle voit et partage avec sa mère. A 4 ans, le désir de montrer sa connaissance du texte domine. Il est intéressant de comparer les deux clôtures des échanges.

En $\mathrm{C} 1$, après une formulation conclusive, «alors voilà ! », Jeanne, n'attendant pas la réaction de sa mère, se met à commenter la $3^{\text {ème }}$ de couverture, puis propose d'emblée de raconter une autre histoire : «Je vais te raconter l'histoire de Mimi la souris ». Réaction immédiate de la mère : «Une autre fois, minette !».

En C2, Jeanne termine par le texte exact de l'histoire, se tourne vers sa mère, sourit largement et attend sa réaction (son évaluation ?) que la mère s'empresse de donner: «Bravo, Jeanne, c'est vraiment très bien! ».

Cette différence et l'évolution de la narration chez Jeanne porte-t-elle des traces de sa scolarisation à l'école maternelle? On peut en faire l'hypothèse. On notera, par exemple, que l'abandon en C2 d'une focalisation sur l'image au profit du texte a modifié nettement la prosodie du récit de Jeanne. La fluidité et la linéarité du discours qui caractérisent $\mathrm{C} 2$, marquent un apprentissage d'une norme du raconté «comme il faut» et tranchent avec les pauses, les retours en arrière caractéristique de $\mathrm{C} 1$. Le ton employé en $\mathrm{C} 2$ est bien celui d'une posture de narratrice avec une mise en scène de soi comme sujet racontant dont on peut penser qu'elle est empruntée aux adultes (la mère ? la maîtresse ?). En C1, Jeanne est dans une importante activité d'exploration de l'histoire. En $\mathrm{C} 2$, elle investit vraiment le rôle de conteuse. Si l'école a eu sa part d'influence sur cette évolution de la narration chez Jeanne, c'est qu'elle a joué son rôle de transmission de modèles de discours et, particulièrement pour la narration, de modèles qui conduisent à l'appropriation de pratiques littéraciées (Pouëch, 2001). 
Mais, dans le même temps, on aimerait que cette nécessaire appropriation de modèles ne se fasse pas au détriment d'une liberté de prise de parole par l'enfant, tellement inattendue et source d'invention et de plaisir. Finalement, en $\mathrm{C} 1$, Jeanne mène diverses activités autour de son exploration de l'histoire. Elle observe et commente les illustrations, pose des questions sur ce qu'elle ne comprend pas, extrapole en commentant des éléments de l'histoire qu'elle sort de leur contexte, rentre en interaction avec les personnages, etc. Autant de détournements (du point de vue de l'adulte) du texte d'origine qui devient le lieu d'un foisonnement de jeux de langage. Mais on doit sans doute au genre narratif lui-même le double visage d'être tout à la fois le moyen d'une libre expression de soi et celui d'une activité langagière socialement identifiée et majeure. L'expérience enfantine du récit donne à voir, dans son appropriation, ce double visage et c'est pourquoi l'enfant est un merveilleux informateur pour le chercheur.

\section{REFERENCES}

DELAMOTTE Régine (1997) Reprises, impositions, résistances et autres inventions : enfants de quatre ans et adultes face au récit, Le langage à l'école maternelle, CALAP, Fascicule 14, Paris René Descartes, 59-79.

DELAMOTTE Régine (2004) Ce que les récits enfantins nous apprennent sur le récit et le reste, Enfants et récits, Chapitre 1, Lille, Septentrion, 13-41.

DELAMOTTE Régine, AKINCI Mehmet-Ali (dirs) (2012) Récits d'enfants. Développement, genre, contexte, Rouen, PURH.

FRANÇOIS Frédéric (1990) Plaisir et structure. L'enfant «créateur»: le texte des adultes, sa reformulation, sa modification par l'enfant, Littérature, société et droits de l'enfant, Paris, ENFANCE, $1-2,176-186$.

FRANÇOIS Frédéric (1993) Pratiques de l'oral, Paris, Nathan.

FRANÇOIS Frédéric (1997) Langue, dessin, langage », CALAP, Fascicule 14, Le langage à l'école maternelle, Paris René Descartes, 81-102.

FRANÇOIS Frédéric (2004) Structure et fantaisie ou qu'est-ce que fait un enfant de 3 / 6 ans lorsqu'il raconte ?, Enfants et récits, Lille, Septentrion, 71-97.

FREIER Catherine, GROSSMANN Francis, PONS Martine (2005) Littératie familiale et lectures partagées, Barré-de-Miniac Christine et alii (dirs) La littératie : conceptions théoriques et pratiques d'enseignement de la lecture-écriture, Paris, l'Harmattan, 247-261.

GARITTE Catherine (1998) Le développement de la conversation chez l'enfant, Paris, Bruxelles, De Boeck Université.

GROSSMANN Francis (1996) Enfance de la lecture. Manières de faire, manières de lire à l'école maternelle, Berne, Peter Lang. 
HUDELOT Christian (1989) Circulation et restructuration de la référence dans un dialogue adulteenfant, Calap, Fascicule 6, Continuité et diversité dans des dialogues d'enfants, Paris René Descartes, 79-92.

MORGENSTERN Aliyah (2009) L'enfant dans la langue, Paris, Presses Sorbonne Nouvelle.

NALOUTI Mongia (1998) Aspects de l'évolution d'une enfant de 5 ans 8 mois à 9 ans 2 mois. Organisation narrative. Références et formes discursives, Thèse de doctorat, Université de Paris 3.

NONNON Elisabeth (2000) Ce que le récit oral peut nous dire sur le récit, REPERES, 21, Diversité narrative, Paris, INRP, 23-52.

PLUMET Marie-Hélène (2008) Développement des interactions sociales et théorie de l'esprit: fonctionnements et dysfonctionnements, Interactions, acquisitions et apprentissages, de Weck Geneviève et Salazar Orvig Anne (éds), Neuchâtel, TRANEL, 49, 9-28.

POUËCH Françoise (2001) Regard sur des récits d'enfants, CALAP, Fascicule 21-22, Récit, Dialogue et commentaire chez de jeunes enfants, Paris René Descartes, 217-249.

REUTER Yves (2005) L'analyse du récit, Paris, Colin, 128 pages.

REUTER Yves, TAUVERON Catherine (coords) (2000) Diversité narrative, REPERES, 21, Paris, INRP.

SALAGNAC Nathalie (2007) Comment évaluer les compétences narratives des enfants à partir de la diversité des conduites narratives?», Les Cahiers THEODILE, 7, Villeneuve d'Ascq, Université Charles-de-Gaulle Lille 3, 81-96. 